\title{
LINEAR TORIC FIBRATIONS
}

\author{
SANDRA DI ROCCO
}

\section{CONTENTS}

Introduction $\quad 1$

Conventions 2

1. Introduction to toric fibrations 2

Combinatorial characterization 2

Historical Remark $\quad 4$

2. Toric discriminants and toric fibrations 4

Historical remarks. $\quad 4$

A first characterization $\quad 5$

3. Toric fibrations and adjunction theory 8

$\begin{array}{lr}\text { Second characterization. } & 9\end{array}$

References $\quad 11$

Introduction. The purpose of this series of lectures is to introduce the notion of toric fibration and to give their geometrical and combinatorial characterizations. A toric fibration $X \rightarrow Y$ is associated to a surjective map of lattices $\pi: M \rightarrow \Lambda$. A polarized toric fibration defines a polytope in $M$ called a Cayley sum and denoted by Cayley $\left(R_{0}, \ldots, R_{t}\right)_{\pi, Y}$. Our aim is to illustrate how classical notions in projective geometry are captured by certain properties of the associated Cayley sums.

Lecture 1 will be devoted to define these concepts and to give the most relevant examples. In the following two lectures we will present two characterizations of Cayley sums. In both cases there are reach and interesting connections with classical projective geometry.

Characterization 1. (see section 2). Let $P$ be a smooth polytope. The following assertions are equivalent:

(a) $P=$ Cayley $_{\pi, Y}\left(R_{0}, \ldots, R_{t}\right)$ with $t \geqslant \max \left(2, \frac{n+1}{2}\right)$.

(b) $\sum_{\emptyset \neq F \prec P}(-1)^{\operatorname{codim}(F)}(\operatorname{dim}(F)+1) \operatorname{Vol}(F)=0$.

(c) $\operatorname{codim}\left(X_{P}^{*}\right)>1$.

Characterization 2. (see section 3). Let $P$ be a smooth polytope. The following assertions are equivalent:

(a) $\operatorname{codeg}(P) \geqslant(n+3) / 2$.

Date: June 2013. 
(b) $P$ is isomorphic to a Cayley sum Cayley $\left(R_{0}, \ldots, R_{t}\right)_{\pi, Y}$ where $t+1=\operatorname{codeg}(P)$ with $k>\frac{n}{2}$.

(c) $\mu\left(\mathscr{L}_{P}\right)=\tau\left(\mathscr{L}_{P}\right) \geqslant(n+3) / 2$.

Conventions. We assume basic knowledge of toric geometry and refer to [EW, FU, ODA] for the necessary background on toric varieties. Throughout this paper, we work over the field of complex numbers $\mathbb{C}$. Toric varieties, $X$, are always normal and thus defined by a fan $\Sigma_{X} \subset N$. By $\Sigma_{X}(n)$ we will denote the collection of $n$-dimensional cones of $\Sigma_{X}$.

Let $P \subset \mathbb{R}^{n}$ be a lattice polytope of dimension $n$. Consider the graded semigroup $\Pi_{P}$ generated by $(\{1\} \times P) \cap\left(\mathbb{N} \times \mathbb{Z}^{n}\right)$. The polarized variety $\left(\operatorname{Proj}\left(\mathbb{C}\left[\Pi_{P}\right]\right), \mathscr{O}(1)\right)$ is a toric variety associated to the polytope $P$. It will be sometime denoted by $\left(X_{P}, L_{P}\right)$. Notice that the toric variety $X_{P}$ is defined by the (inner) normal fan of $P$. Viceversa the symbol $P_{(X, L)}$ will denote the lattice polytope associated to a polarized toric variety $(X, L)$.

The symbol $\Delta_{n}$ denotes the smooth (unimodular) simplex of dimension $n$. Recall that an $n$-dimensional polytope is simple if through every vertex pass exactly $n$ edges. A lattice polytope is smooth if it is simple and the primitive vectors of the edges through every vertex form a lattice basis. Smooth polytopes are associates to smooth projective varieties. Simple polytopes are associated to $\mathbb{Q}$-factorial projective varieties.

\section{INTRODUCTION TO TORIC FIBRATIONS}

Definition 1.1. A toric fibration is a surjective flat map $f: X \rightarrow Y$ with connected fibres where

(a) $X$ is a toric variety

(b) $Y$ is a normal algebraic variety

(c) $\operatorname{dim}(Y)<\operatorname{dim}(X)$.

Remark 1.2. Observe that if $f: X \rightarrow Y$ is a toric fibration then $Y$ and a general fiber $F$ are also toric varieties. Moreover if $X$ is smooth, respectively $\mathbb{Q}$-factorial then so is $Y$ and $F$.

Combinatorial characterization. A toric fibration has the following combinatorial characterization (see [EW, Chapter VI] for further details). Let $X=X_{\Sigma}$, where $\Sigma \subset N \cong \mathbb{Z}^{n}$, be a toric variety of dimension $n$ and let $i: \Delta \hookrightarrow N$ a sublattice.

Proposition 1.3. [EW] The inclusion $i$ induces a toric fibration if and only if:

(a) $\Delta$ is a primitive lattice, i.e. $(\Delta \otimes \mathbb{R}) \cap N=\Delta$.

(b) For every $\sigma \in \Sigma(n), \sigma=\tau+\eta$, where $\tau \in \Delta$ and $\eta \cap \Delta=\{0\}$ (i.e. $\Sigma$ is a split fan).

We briefly outline the construction. The projection $\pi: N \rightarrow N / \Delta$ induces a map of fans $\Sigma \rightarrow \pi(\Sigma)$ and thus a map of toric varieties $f: X \rightarrow Y$. The general fiber $F$ is a toric variety defined by the fan $\Sigma_{F}=\{\sigma \in \Sigma \cap \Delta\}$.

When the toric variety $X$ in a toric fibration is polarized by an ample line bundle $L$ we will call the pair $(f: X \rightarrow Y, L)$ a polarized toric fibration. Observe that the polarized toric varieties $(X, L)$ and $\left(F,\left.L\right|_{F}\right)$, for a general fiber $F$, define lattice polytopes $P_{(X, L)}, P_{\left(F,\left.L\right|_{F}\right)}$. The polytope $P_{(X, L)}$ is in fact a "twisted sum" of a finite number of lattice polytopes fibering over $P_{\left(F,\left.L\right|_{F}\right)}$. 
Definition 1.4. Let $R_{0}, \ldots, R_{k} \subset \Delta$ be polytopes. Let $\pi: M \rightarrow \Lambda$ be a surjective map of lattices such that $\pi\left(R_{i}\right)=v_{i}$ and the $v_{0}, \cdots, v_{k}$ are distinct vertices of $\operatorname{Conv}\left(v_{0}, \ldots, v_{k}\right)$. We will call $a$ Cayley $\pi$-twisted sum (or simply a Cayley sum) of $R_{0}, \ldots, R_{k}$ a polytope which is affinely isomorphic to $\operatorname{Conv}\left(R_{0}, \ldots, R_{k}\right)$. We will denote it by:

$$
\left[R_{0} \star \ldots \star R_{k}\right]_{\pi}
$$

If the polytopes $R_{i}$ are additionally normally equivalent, i.e. they define the same normal fan $\Sigma_{Y}$, we will denote the Cayley sum by:

$$
\text { Cayley }\left(R_{0}, \ldots, R_{k}\right)_{(\pi, Y)} \text {. }
$$

These are the polytopes that are associated to a polarized toric fibration. Consider a sublattice $i: \Delta \hookrightarrow N$ and the dual lattice surjection $\pi: M \rightarrow \Lambda$.

Proposition 1.5. [CDR08] The sublattice $i: \Delta \hookrightarrow N$ induces a polarized toric fibration $(f: X \rightarrow Y, L)$ if and only if $P_{(X, L)}=$ Cayley $\left(R_{0}, \ldots, R_{k}\right)_{(\pi, Y)}$ for some normally equivalent polytopes $R_{0}, \ldots, R_{k}$.

The polarized general fiber $\left(F,\left.L\right|_{F}\right)$ corresponds to the polarized toric variety associated to the polytope $P_{\left(F,\left.L\right|_{F}\right)}=\operatorname{Conv}\left(v_{0}, \ldots, v_{k}\right)$ and the polytopes $R_{0}, \cdots, R_{k}$ define the embeddings of the invariant sections polarized by the restrictions of $L$.

Example 1.6. Consider the Hirzebruch surface $\mathbb{F}_{1}=B l_{p}\left(\mathbb{P}^{2}\right)=\mathbb{P}\left(\mathscr{O}_{\mathbb{P}^{1}} \oplus \mathscr{O}_{\mathbb{P}^{1}}(1)\right)$ polarized by the tautological line bundle $\xi=2 \phi^{*}\left(\mathscr{O}_{\mathbb{P}^{2}}(1)\right)-E$ where $\phi$ is the blow-up map and $E$ the exceptional divisor. The associated polytope is $P=\operatorname{Cayley}\left(\Delta_{1}, 2 \Delta_{1}\right)$.

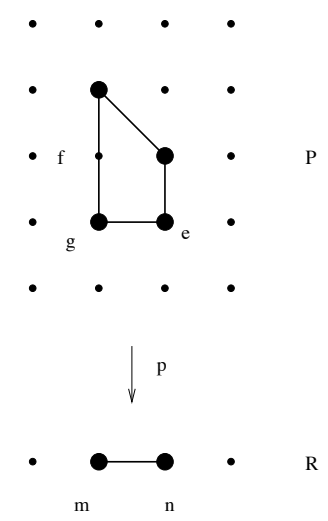

FIGURE 1. The Hirzebruch surface $\mathbb{P}\left(\mathscr{O}_{\mathbb{P}^{1}} \oplus \mathscr{O}_{\mathbb{P}^{1}}(1)\right)$

Example 1.7. More generally:

- when $\pi(P)=\Delta_{t}$ the polytope Cayley $\left(R_{0}, \ldots, R_{k}\right)_{(\pi, Y)}$ defines the variety $\mathbb{P}\left(L_{0} \oplus \ldots \oplus\right.$ $L_{k}$ ), where the $L_{i}$ are ample line bundles on the toric variety $Y$, polarized by the tautological bundle $\xi$. In particular $\left.L\right|_{F}=\mathscr{O}_{\mathbb{P} t}(1)$.

- When $\pi(P)$ is a simplex (not necessarily smooth) Cayley $\left(R_{0}, \ldots, R_{k}\right)_{(\pi, Y)}$ defines a Mori-type fibration. A fibration whose general fiber has Picard rank one. 
- When $\pi(P)=s \Delta_{t}$ then again the variety has the structure of a $\mathbb{P}^{t}$-fibration whose general fiber $F$ is embedded via an $s$-Veronese embedding: $\left(F,\left.L\right|_{F}\right)=\left(\mathbb{P}^{t}, \mathscr{O}_{\mathbb{P}^{t}}(s)\right)$.

For general Cayley sums, $\left[R_{0} \star \ldots \star R_{k}\right]_{\pi}$, one has the following geometrical interpretation. Let $(X, L)$ be the associated polarized toric variety and let $Y$ be the toric variety defined by the Minkowski sum $R_{0}+\ldots+R_{k}$. The fan defining $Y$ is a refinement of the normal fan of $R_{i}$ for $i=0, \ldots, k$. Consider the associated birational maps $\phi_{i}: Y \rightarrow Y_{i}$, where $\left(Y_{i}, L_{i}\right)$ is the polarized toric variety defined by the polytope $R_{i}$. The line bundles $H_{i}=\phi_{i}^{*}\left(L_{i}\right)$ are nef line bundles on $Y$. Denote by the same symbol the maps of fans $\phi_{i}: \Sigma_{Y} \rightarrow \Sigma_{Y_{i}}$. Define then the fan:

$$
\Sigma_{Z}:\left\{\phi_{i}^{-1}\left(\sigma_{j}\right) \times \eta_{l}, \text { for all } \sigma_{j} \in \Sigma_{Y_{i}}, \eta_{l} \in \Sigma_{\Delta}\right\}
$$

where $\Lambda=\operatorname{Conv}\left(v_{0}, \ldots, v_{k}\right)$. It is a refinement of $\Sigma_{X}$ and thus the defining variety $Z$ is birational to $X$. Moreover it is a split fan and thus it defines a toric fibration $f: Z \rightarrow Y$. The Cayley sum $\left[R_{0} \star \ldots \star R_{k}\right]_{\pi}$ is the polytope defined by the nef line bundle $\phi^{*}(L)$, and the polytopes $R_{i}$ are the polytopes defined by the nef line bundles $H_{i}$ on the invariant sections.

Historical Remark. The definition of a Cayley polytope originated by what is "classically" referred to as the Cayley trick. We first recall the definition of Resultant and Discriminant. Let $f_{1}(x), \ldots, f_{n}(x)$ be a system of $n$ polynomials in $n$ variables $x=\left(x_{1}, \ldots, x_{n}\right)$ supported on $A \subset \mathbb{Z}^{n}$. This means that $f_{i}=\Pi_{a_{j} \in A} c_{j} x^{a_{j}}$. The resultant (of $A$ ), $R_{A}\left(c_{j}\right)$, is a polynomial in the coefficients $c_{j}$, which vanishes whenever the corresponding polynomials have a common zero.

The discriminant of a finite subset $A, \Delta_{\mathscr{A}}$, is also a polynomial $\Delta_{\mathscr{A}}\left(c_{j}\right)$ in the variables $c_{j} \in A$ which vanishes whenever the corresponding polynomial has a multiple root.

Theorem 1.8. [GKZ]/Cayley Trick] The A-resultant of the system $f_{1}, \ldots, f_{n}$ equals the Adiscriminant of the polynomial:

$$
p(x, y)=f_{i}(x)+\sum_{2}^{n} y_{i-1} f_{i}(x) .
$$

Let $R_{i}=N\left(f_{i}\right) \subset \mathbb{R}^{n}$ be the Newton polytopes of the polynomials $f_{i}$. The Newton polytope of the polynomial $p(x, y)$ is the Cayley sum $\left[R_{1} \star \ldots \star R_{n}\right]_{\pi}$, where $\pi: \mathbb{R}^{2 n-1} \rightarrow \mathbb{R}^{n-1}$ is the natural projection such that $\pi\left(\left[R_{1} \star \ldots \star R_{n}\right]_{\pi}\right)=\Delta_{n-1}$.

\section{TORIC DISCRIMINANTS AND TORIC FIBRATIONS}

Historical remarks. The term "discriminant" is well known in relation with low degree equations or ordinary differential equations and it has a well defined geometrical meaning naturally connected to the way our vision grasps geometrical shapes. The way our eye sees a 3-dimensional object is through its 2-dimensional boundary. More precisely if we place our eye at infinity on the $x$-axis and we assume that the boundary is defined (even locally) by a polynomial equation $f(x, y, z)=0$, what we really see is a curve defined by the points of contact of the rays originating at our eye and which are tangent to the boundary. This curve is obtained by eliminating the variable $x$ from the system $\{f(x, y, z), \partial f(x, y, z) / \partial x\}$. 
The defining equation of the curve is called the discriminant of $f$ (with respect to the variable $x)$.

More generally let $\mathscr{A}=\left\{a_{0}, \ldots, a_{m}\right\}$ be a subset of $\mathbb{Z}^{n}$. The discriminant of $\mathscr{A}$ (when it exists) is an irreducible homogeneous polynomial $\Delta_{\mathscr{A}}\left(c_{0}, \ldots, c_{m}\right)$ vanishing when a general Laurent polynomial supported on $\mathscr{A}, f(x)=\sum_{a_{i} \in \mathscr{A}} c_{i} x^{a_{i}}$, has at least one multiple root in the torus $\left(\mathbb{C}^{*}\right)^{n}$. Geometrically, the zero-locus of the discriminant is an irreducible algebraic variety of codimension one in the dual projective space $\mathbb{P}^{m *}$, called the dual variety of the embedding $X_{\mathscr{A}} \subset \mathbb{P}^{m}$. For a fixed embedding $i: X \hookrightarrow \mathbb{P}^{m}$ of an $n$-dimensional algebraic variety, the dual variety is the Zariski-closure of all the hyperplanes $H \subset \mathbb{P}^{m}$ tangent to $X$ at some non singular point. We can speak of a discriminant only when the dual variety has codimension one. Embeddings whose dual variety has higher codimension are called defective and the discriminant is set to be 1 . Finding formulas for $\Delta_{\mathscr{A}}$ and classifying the point configurations $\mathscr{A}$ with discriminant 1 (defect toric embeddings) is a long standing problem in combinatorics, toric geometry and algebraic geometry. Dickenstein-Strumfels, [DS02], characterized the cases when $m=n+2$, Cattani-Curran, [CC07] extended the classification to $m=n+3, n+4$. In these cases the corresponding embedding is possibly very singular and the methods used are purely combinatorial. In [DiR06] and [CDR08] we completely characterize the case when $\operatorname{Conv}(\mathscr{A})$ is smooth or simple, correspondingly when the toric embedding is smooth or Q-factorial:

Theorem 2.1. Let $\left|\mathscr{A} \cap \mathbb{Z}^{n}\right|=\left|P_{\mathscr{A}} \cap \mathbb{Z}^{n}\right|$ and assume $X_{\mathscr{A}}$ is $\mathbb{Q}$-factorial. Then the configuration is defective if and only if $X_{\mathscr{A}}$ is a Mori-fibration (it admits a surjective flat map onto a $\mathbb{Q}$-factorial toric variety whose generic fibre is reduced and has Picard number one). Equivalently $P=\operatorname{Conv}(\mathscr{A})$ is a Cayley sum Cayley $\left(R_{0}, \ldots, R_{t}\right)_{(\pi, Y)} \subset \mathbb{R}^{n+t}$, where $\pi(P)$ is a simplex (not necessarily standard) in $\mathbb{R}^{t}$ and $R_{0}, \ldots, R_{t}$ are normally equivalent polynomials. If moreover $X_{\mathscr{A}}$ is non singular then $\left(X_{\mathscr{A}}, L_{\mathscr{A}}\right)=\left(\mathbb{P}\left(L_{0} \oplus \cdots \oplus L_{t}\right), \xi\right)$.

A first characterization. We start with giving a more algebro-geometrical definition of the dual variety. Multiple roots of fixed multiplicity $k$ correspond to hyperplanes tangent "to the order $k$." Consider an embedding $i: X \hookrightarrow \mathbb{P}^{m}$ given by the global sections of $\mathscr{L}=i^{*}\left(\mathscr{O}_{\mathbb{P} m}(1)\right)$. For any smooth point $x$ of the embedded variety let:

$$
j e t_{x}^{k}: H^{0}(X, \mathscr{L}) \rightarrow H^{0}\left(X, \mathscr{L} \otimes \mathscr{O}_{X} / \mathfrak{m}_{x}^{k+1}\right)
$$

be the map assigning to $s$ in $H^{0}(X, \mathscr{L})$ the tuple $\left(s(x), \ldots,\left(\partial^{t} s / \partial \underline{x}^{t}\right)(x), \ldots\right)_{t \leqslant k}$ where $\underline{x}=$ $\left(x_{1}, \ldots, x_{n}\right)$ is a local system of coordinates around $x$. The $k$-th osculating space at $x$ is defined as $\mathbb{O} s c_{x}^{k}=\mathbb{P}\left(\operatorname{Im}\left(\right.\right.$ jet $\left.\left._{x}^{k}\right)\right)$. As the map jet ${ }_{x}^{1}$ is surjective, the first osculating space is isomorphic to $\mathbb{P}^{n}$ and classically called the projective tangent space. A hyperplane $H \subset \mathbb{P}^{m}$ is tangent at $x$ to the order $k$ if it contains the $k$-th osculating space. One can then define the $k$-th order dual variety $X^{k}$ as:

$$
X^{k}=\overline{\left\{H \in \mathbb{P}^{*} \text { tangent to the order } k \text { to } X \text { at some non singular point }\right\}} .
$$

General properties of the higher order dual variety have been extensively studied by S. Kleiman and R. Piene and were described mainly in the case of scrolls. 
Consider the projections $\pi_{i}: X \times X \rightarrow X$ and the ideal sheaf of the diagonal $\mathscr{I}_{\Delta_{X}}$. The $k$-the jet bundle of $\mathscr{L}$ is defined as $J_{k}(\mathscr{L})=\pi_{2 *}\left(\phi_{1}^{*}(\mathscr{L}) \otimes\left(\mathscr{O}_{X \times X} / \mathscr{I}_{\Delta_{X}}^{k+1}\right)\right)$. When the variety $X$ is smooth $J_{k}(\mathscr{L})$ is a vector bundle of rank $\left(\begin{array}{c}n+k \\ n\end{array}\right)$. It is important to note that when the map $j e t_{x}^{k}$ is surjective for all smooth points $x$, then properties of $X^{k}$ can be related to vanishing of Chern classes of the associated $k$-th jet bundle, $J_{k}(\mathscr{L})$,

Definition 2.2. A line bundle $\mathscr{L}$ on $X$ is called $k$-spanned at $x$ if the map jet ${ }_{x}^{k}$ is surjective. It is called $k$-spanned if it is $k$-spanned at every point.

For general $k$-spanned $n$-dimensional embeddings in $\mathbb{P}^{m}$ the codimension of $X^{k}$ is $\left(\begin{array}{c}n+k \\ k\end{array}\right)-n$ and the degree is given by a multiple of the top Chern class $c_{n}\left(J_{k}(\mathscr{L})\right)$. When the codimension is higher the embedding is said to be $k$-th defective.

Lemma 2.3. [LM00, DDRP12] Let $X$ be a smooth variety and $\mathscr{L}$ a $k$-spanned line bundle. Then codim $\left(X^{k}\right)>\left(\begin{array}{c}n+k \\ k\end{array}\right)-n$ if and only if $c_{n}\left(J_{k}(\mathscr{L})\right)=0$. Moreover $\operatorname{deg}\left(X_{A}^{k}\right)=$ $n(\mathscr{A})_{k} c_{n}\left(\left(J_{k}(\mathscr{L})\right)=0\right.$, where $n_{k}(\mathscr{A})$ is an integer and it is equal to one for $k=1$.

In the smooth case one can compute the top Chern class of the jet bundles from the following diagram:

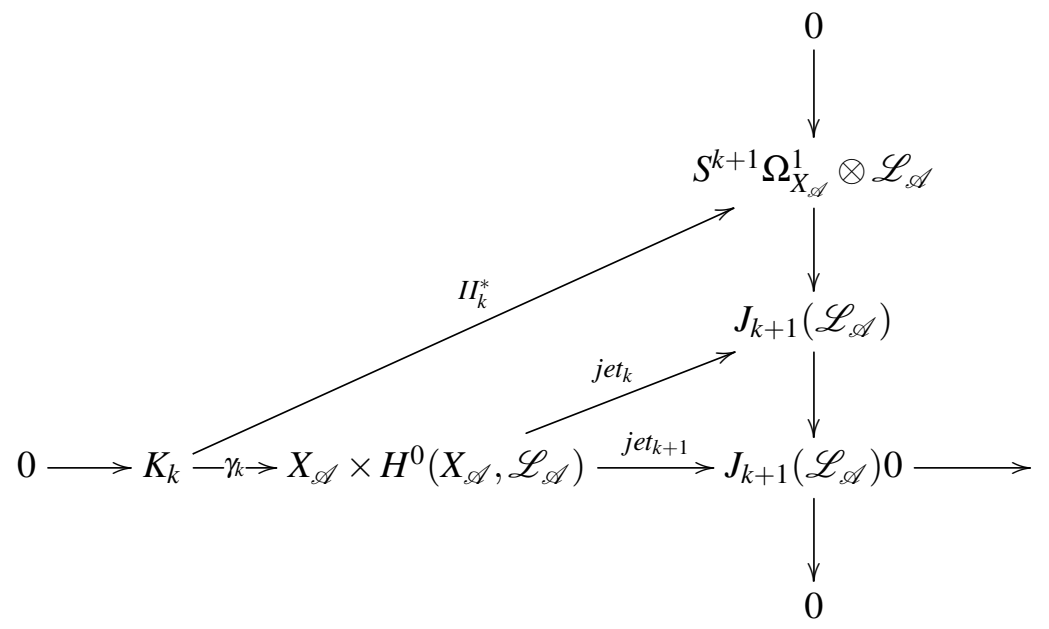

where the map $I I_{k}^{*}$ is the dual of the $k$-the fundamental form. See [L94, GH79] for more details. Taking the dual of $\gamma_{k}$ and projectivizing the corresponding vector bundles one gets a projection:

$$
\alpha_{k}: \mathbb{P}\left(K_{k}^{*}\right) \rightarrow \mathbb{P}\left(H^{0}(X, L)^{*}\right), X_{\mathscr{A}}^{k}=\operatorname{Im}\left(\alpha_{k}\right), n(\mathscr{A})_{k}=\operatorname{deg}\left(\alpha_{k}\right) .
$$

In the general case of singular toric varieties the jet bundles are no longer locally free and thus it is not possible to use Chern-classes techniques. Using combinatorics and convex geometry one can generalize the characterization above as follows.

Proposition 2.4. [GKZ, DiR06, MT11] Let $\left(X_{\mathscr{A}}, L_{\mathscr{A}}\right)$ be a polarized toric variety associated to the polytope $P_{\mathscr{A}}$. Set

$$
\delta_{i}=\sum_{\emptyset \neq F \prec P}(-1)^{\operatorname{codim}(F)}\left\{\left(\begin{array}{c}
\operatorname{dim}(F)+1 \\
i
\end{array}\right)+\left((-1)^{i-1}(i-1)\right\} \operatorname{Vol}(F) \operatorname{Eu}(V(F)) .\right.
$$


Then $\operatorname{codim}\left(X_{\mathscr{A}}^{*}\right)=r=\min \left\{i, \delta_{i} \neq 0\right\}$ and $\operatorname{deg}\left(X_{\mathscr{A}}^{*}\right)=\delta_{r}$.

The function $\mathrm{Eu}: X_{A} \rightarrow \mathbb{Z}$ assigns an integer to all invariant sub varieties. This value is different from 1 only when the variety is singular. In particular, when $X_{\mathscr{A}}$ is smooth we have:

$$
\operatorname{codim}\left(X_{A}^{*}\right)>1 \Leftrightarrow \sum_{\emptyset \neq F \prec P}(-1)^{\operatorname{codim}(F)}(\operatorname{dim}(F)+1) \operatorname{Vol}(F)=0
$$

In fact in the smooth case one can prove directly:

$$
c_{n}\left(J_{1}\left(\mathscr{L}_{\mathscr{A}}\right)\right)=\sum_{\emptyset \neq F \prec P}(-1)^{\operatorname{codim}(F)}(\operatorname{dim}(F)+1) \operatorname{Vol}(F)
$$

This gives the first characterization of certain Cayley sums and toric fibrations:

Proposition 2.5. [DiR06] Let $P \subset \mathbb{R}^{n}$ be a smooth n-dimensional polytope. The following are equivalent:

(a) $P=$ Cayley $_{\pi, Y}\left(R_{0}, \ldots, R_{t}\right)$ with $t \geqslant \max \left(2, \frac{n+1}{2}\right)$.

(b) $\sum_{\emptyset \neq F \prec P}(-1)^{\operatorname{codim}(F)}(\operatorname{dim}(F)+1) \operatorname{Vol}(F)=0$.

(c) $X_{P}=\mathbb{P}\left(L_{0} \oplus \ldots \oplus L_{t}\right)$, for line bundles $L_{i}$ on a smooth toric variety $Y$ and such that $t \geqslant \max \left(2, \frac{n+1}{2}\right)$.

(d) $c_{n}\left(J_{1}\left(\mathscr{L}_{P}\right)\right)=0$.

Let $\mathscr{A}_{1}, \mathscr{A}_{2}, \ldots, \mathscr{A}_{n}$ be subsets in $\mathbb{Z}^{n}$. Consider Laurent polynomials in $n$ unknowns $f_{i}(x)=$ $\sum_{a \in A_{i}} c_{i, a} x^{a}$ (with fixed support $\mathscr{A}_{i}$ ). If the coefficients $c_{i, a}$ are generic then, according to Bernstein's Theorem [1975], the number of solutions of the system $f_{1}(X)=\cdots=f_{n}(x)=0$ in the algebraic torus $\left(\mathbb{C}^{*}\right)^{n}$ equals the mixed volume $M V\left(Q_{1}, Q_{2}, \ldots, Q_{n}\right)$ of the Newton polytopes $Q_{i}=\operatorname{Conv}\left(\mathscr{A}_{i}\right)$ in $\mathbb{R}^{n}$. However, for special choices of the coefficients $c_{i, a}$, two or more of these solutions may come together in $\left(\mathbb{C}^{*}\right)^{n}$ and create a point of higher multiplicity. The conditions under which this happens are encoded in an irreducible polynomial, the mixed discriminant $\Delta_{\mathscr{A}_{1}, \ldots, \mathscr{A}_{n}}$, in the coefficients $c_{i, a}$, whose zero locus is the variety of ill-posed systems. For a single polynomial this definition coincides with the discriminant and the dual variety introduced above. One defines the discriminantal variety as the closure of the locus of coefficients $c_{i, a}$ for which the system has a non-degenerate multiple root. If the discriminantal variety is a hypersurface, we define the mixed discriminant of the system to be the unique (up to sign) irreducible polynomial $\Delta_{\mathscr{A}_{1}, \ldots, \mathscr{A}_{n}}$ with integer coefficients in the unknowns $c_{i, a}$ which defines it. Otherwise we say that the system is defective and set $\Delta_{\mathscr{A}_{1}, \ldots, \mathscr{A}_{n}}=1$. The support configurations $\mathscr{A}_{1}, \ldots, \mathscr{A}_{n}$ can be assembled to form a Cayley sum, $\operatorname{Conv}(\mathscr{A})$.

Theorem 2.6. [CCDDRS] The mixed discriminant equals the $\mathscr{A}$-discriminant of the Cayley matrix: $\Delta_{\mathscr{A}_{1}, \ldots, \mathscr{A}_{n}}=\Delta_{\mathscr{A}}$.

As for higher order discriminants a classification even in the smooth case is still open. In [DDRP12] a classification for dimension 3 and $k=2$ is given.

Theorem 2.7. [DDRP12] Let $(X, \mathscr{L})$ be a smooth, 2-regular toric threefold embedding corresponding to a lattice polytope $P$. Then the second dual variety has codimension higher than expected if and only if $(X, \mathscr{L})=\left(\mathbb{P}^{3}, \mathscr{O}(2)\right)$, Moreover: 


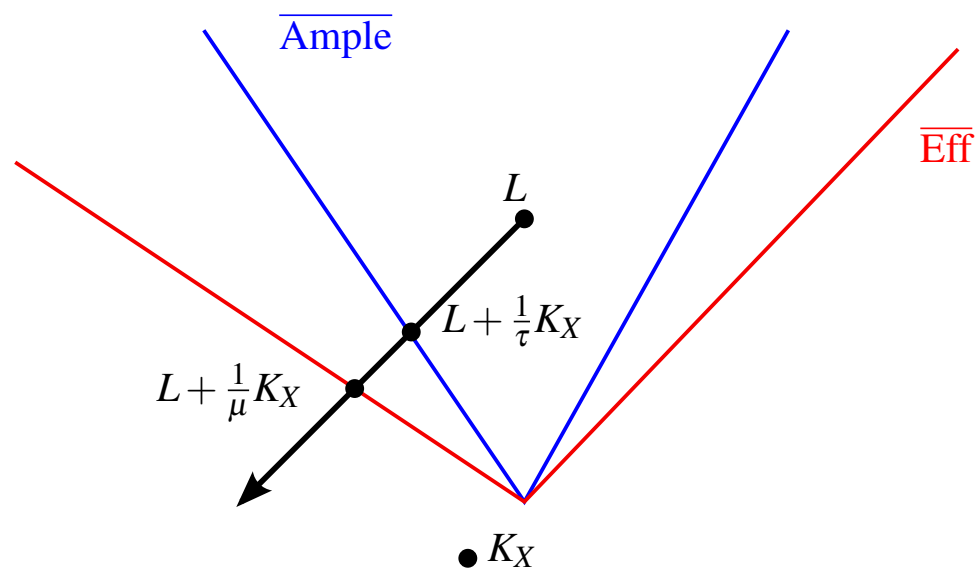

FIGURE 2. Illustrating $\mu(\mathscr{L})$ and $\tau(\mathscr{L})$

(1) $\operatorname{deg} X^{2}=120$ if $(X, \mathscr{L})=\left(\mathbb{P}^{3}, \mathscr{O}(3)\right)$.

(2) $\operatorname{deg} X^{2}=6(8(a+b+c)-7)$ if $(X, \mathscr{L})=\left(\mathbb{P}\left(\mathscr{O}_{\mathbb{P}^{1}}(a), \mathscr{O}_{\mathbb{P}^{1}}(b), \mathscr{O}_{\mathbb{P}^{1}}(c)\right), 2 \xi\right)$, where $a, b, c \geq 1$ and $\xi$ denotes the tautological line bundle.

(3) In all other cases, $\operatorname{deg}_{2} X^{2}=t\left(62 V-57 \mathscr{F}+28 \mathscr{E}-8 \mathscr{V}+58 V_{1}+51 \mathscr{F}_{1}+20 \mathscr{E} 1\right)$ where $V, \mathscr{F}, \mathscr{E}$ (respectively. $V_{1}, \mathscr{F}_{1}, \mathscr{E}_{1}$ ) denote the volume, area of facets, length of edges of $P$ (respectively. of the convex hull of $\operatorname{int}(P)), \mathscr{V}$ is the number of vertices of $P$ and $t$ is an integer depending on the embedding.

In particular note that the property $c_{2}\left(J_{2}(\mathscr{L})\right)=0$ characterizes only unimodular simplices.

\section{TORIC FIBRATIONS AND ADJUNCTION THEORY}

The classification of projective algebraic varieties is a central problem in Algebraic Geometry dating back to the early nineteenth century. The way one can realistically carry out a classification theory is through invariants, such as the degree, genus, Hilbert polynomial. Modern adjunction theory and Mori theory are the basis for major advances in this area.

Let $(X, \mathscr{L})$ be a polarized $n$-dimensional variety. Assume that $X$ is Gorenstein (the canonical class $K_{X}$ is Cartier). The two key invariants occurring in classification theory, see [Fuj90], are the effective log threshold $\mu(\mathscr{L})$ and the nef value $\tau(\mathscr{L})$, defined as:

$$
\mu(\mathscr{L}):=\sup \left\{s \in \mathbb{Q}: \operatorname{dim}\left(H^{0}\left(K_{X}+s \mathscr{L}\right)\right)=0\right\}, \tau(\mathscr{L}):=\min \left\{s \in \mathbb{R}: K_{X}+s \mathscr{L} \text { is nef }\right\} .
$$

These are both rational numbers $\leqslant n+1$ (Kawamata proved it for $\tau(\mathscr{L})$ and recent advances in the minimal model program establishes it for $\mu(\mathscr{L})$.) These invariants can be visualized as follows.

Traveling from $\mathscr{L}$ in the direction of the vector $K_{X}$ in the Neron-Severi space $\mathrm{NS}(X) \otimes \mathbb{R}$ of divisors, $\mathscr{L}+(1 / \mu(\mathscr{L})) K_{X}$ is the meeting point with the cone of effective divisors $\operatorname{Eff}(X)$ and $\mathscr{L}+(1 / \tau(\mathscr{L})) K_{X}$ is the meeting point with the cone of nef-divisors $\operatorname{Nef}(X)$, see Fig. 2.

In particular when $\tau(\mathscr{L})=\mu(\mathscr{L})$ the line bundle $K_{X}+\tau(\mathscr{L}) \mathscr{L}$ is nef and not big which implies that $\mathscr{L}$ defines a fibration structure on $X$. Algebraic varieties admitting a fibration structure are particularly nice as many of their invariants are induced by corresponding 
invariants on the (lower dimensional) basis and generic fibre. Criteria for a space to be a fibration are therefore highly desirable. Beltrametti-Sommese-Wisniewski conjectured the following.

Conjecture 3.1. [BS94] If $X$ is non singular and $\mu(\mathscr{L})>(n+1) / 2$ then $\mu(\mathscr{L})=\tau(\mathscr{L})$.

Recall that Cayley sums give the associated toric variety the structure of a fibration. As for projective varieties, it is important to be able to detect if a polytope has a Cayley structure using invariants. One invariant, defined by Stanley, which has attracted increasing attention in recent years is the codegree of a lattice polytope:

$$
\operatorname{codeg}(P)=\min \left\{t \in \mathbb{Z}_{>0} \text { such that } t P \text { contains interior lattice points }\right\} .
$$

Via Ehrhart theory one sees that $\operatorname{codeg}(P) \leqslant n+1$ and that $\operatorname{codeg}(P)=n+1$ if and only if $P=\Delta_{n}$. Recently Batyrev and Nill in [BN08] classified polytopes with $\operatorname{codeg}(P)=n$ and conjectured the following.

Conjecture 3.2. [BN08] There is a function $f(n)$ such that any $n$-dimensional polytope $P$ with $\operatorname{codeg}(P) \geqslant f(n)$ decomposes as a Cayley sum of lattice polytopes.

Second characterization. The above conjecture was proven by Haase, Nill and Payne in [HNP09]. They showed that $f(n)$ is at most quadratic in $n$. It is important to observe that, as interior lattice points of $t P$ correspond to global sections of $K_{X}+t \mathscr{L}$ for the associated toric embedding, $\operatorname{codeg}(P)$ can be considered as the integral variant of $\mu(\mathscr{L})$. This observation, techniques from toric Mori theory and adjunction theory led to prove a stronger version of Conjectures 3.1 and 3.2 for smooth polytopes giving a second characterization of Cayley sums.

Theorem 3.3. [DDRP09, DN10] Let $P \subset \mathbb{R}^{n}$ be a smooth $n$-dimensional polytope. Then the following are equivalent.

(a) $\operatorname{codeg}(P) \geqslant(n+3) / 2$.

(b) $P$ is affinity isomorphic to a cayley sum Cayley $\left(R_{0}, \ldots, R_{t}\right)_{\pi, Y}$ where $t+1=\operatorname{codeg}(P)$ with $k>\frac{n}{2}$.

(c) $\mu\left(\mathscr{L}_{P}\right)=\tau\left(\mathscr{L}_{P}\right)$.

(d) $\left(X_{P}, \mathscr{L}_{P}\right)=\mathbb{P}\left(L_{0}, \cdots, L_{t}\right)$ for ample line bundles $L_{i}$ on a non singular toric variety $Y$.

The above conjectures, made independently in two apparently unrelated fields, constitute a beautiful example of the interplay between classical projective (toric) geometry and convex geometry. In view of the results above one could hope that in the toric geometrical setting the conjectures should hold in much more generality. Such general results would represent a significant advance in the classification of projective algebraic varieties and lattice polytopes.

Conjecture 3.4. Let $(X, \mathscr{L})$ be an n-dimensional toric polarized variety (not necessarily smooth or even Gorenstein), then $\mu(\mathscr{L})>(n+1) / 2$ implies that $\mu(\mathscr{L})=\tau(\mathscr{L})$, where the invariants $\mu(\mathscr{L}), \tau(\mathscr{L})$ in the non Gorenstein case are defined using corresponding invariants, $\mu(P), \tau(P)$ of the associated polytope, see below for a definition. 
Conjecture 3.5. If an $n$-dimensional lattice polytope $P$ satisfies $\operatorname{codeg}(P)>(n+1) / 2$, then it decomposes as a Cayley sum of lattice polytopes of dimension at most $2(n+1-\operatorname{codeg}(P))$.

Conjecture 3.4 is a toric version of Conjecture 3.1, extending the statement to possibly singular and non Gorenstein varieties. Conjecture 3.5 states that the function $f(n)$ in Conjecture 3.2 should be equal to $(n+1) / 2$. An important step to prove these conjectures is to define the convex analog of $\mu(\mathscr{L})$.

Let $P \subseteq \mathbb{R}^{n}$ be a rational polytope of dimension $n$. Any such polytope $P$ can be described in a unique minimal way as

$$
P=\left\{x \in \mathbb{R}^{n}:\left\langle a_{i}, x\right\rangle \geqslant b_{i}, i=1, \ldots, m\right\}
$$

where the $a_{i}$ are the rows of an $m \times n$ integer matrix $A$, and $b \in \mathbb{Q}^{m}$.

For any $s \geqslant 0$ we define the adjoint polytope $P^{(s)}$ as

$$
P^{(s)}:=\left\{x \in \mathbb{R}^{n}: A x \geqslant b+s \mathbf{1}\right\},
$$

where $\mathbf{1}=(1, \ldots, 1)^{\mathrm{T}}$.

We call the study of such polytopes $P^{(s)}$ polyhedral adjunction theory.
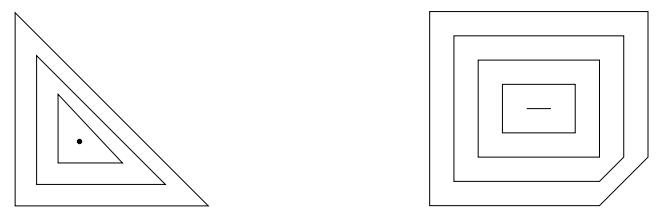

FIGURE 3. Two examples of polyhedral adjunction

Definition 3.6. We define the $\mathbb{Q}$-codegree of $P$ as

$$
\mu(P):=\left(\sup \left\{s>0: P^{(s)} \neq \emptyset\right\}\right)^{-1},
$$

and the core of $P$ to be $\operatorname{core}(P):=P^{(1 / \mu(P))}$.

Notice that the supremum is actually a maximum. Moreover, since $P$ is a rational polytope, $\mu(P)$ is a positive rational number.

One sees that for a lattice polytope $P$

$$
\mu(P) \leqslant \operatorname{codeg}(P) \leqslant n+1,
$$

Definition 3.7. The nef value of $P$ is given as

$$
\tau(P):=\left(\sup \left\{s>0: \mathscr{N}\left(P^{(s)}\right)=\mathscr{N}(P)\right\}\right)^{-1} \in \mathbb{R}_{>0} \cup\{\infty\} .
$$

Note that in contrast to the definition of the $\mathbb{Q}$-codegree, here the supremum is never a maximum.

Fig. 4 illustrates a polytope $P$ with $\tau(P)^{-1}=2, \mu(P)^{-1}=6$. In this case core $(P)$ is an interval.

In [DRHNP13] the precise analogue of Conjecture 3.5 for the $\mathbb{Q}$-codegree is proven. 


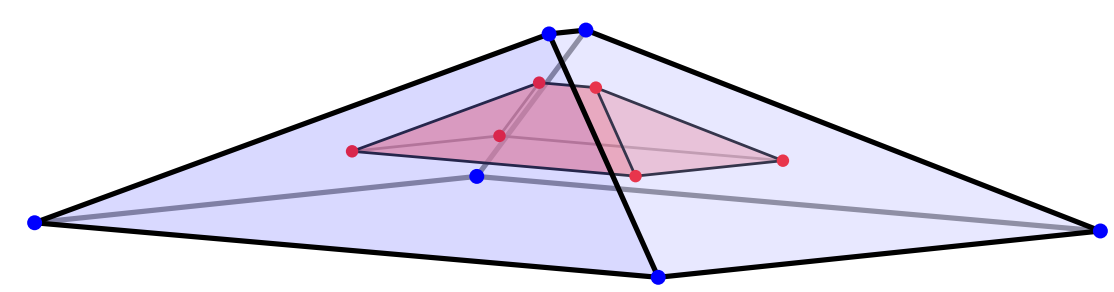

FIgure 4. $P^{(4)} \subseteq P$ for a 3-dimensional lattice polytope $P$

Theorem 3.8. [DRHNP13] Let $P$ be an n-dimensional lattice polytope. If $n$ is odd and $\mu(P)>(n+1) / 2$, or if $n$ is even and $\mu(P) \geqslant(n+1) / 2$, then $P$ is a Cayley polytope.

Results from [DRHNP13] show Conjecture 3.5 in two interesting cases: when $\lceil\mu(P)\rceil=$ $\operatorname{codeg}(P)$ and when the normal fan of $P$ is Gorenstein and $P$ is $\mathbb{Q}$-normal $(\mu(P)=\tau(P))$. This suggests that a proof of Conjecture 3.4 would lead to Conjecture 3.5 if we have good estimates on the difference $\operatorname{codeg}(P)-\mu(P)$. Recent results on the so called "spectrum conjecture" stated by Fujita [1992] and now proved for toric varieties by A. Paffenholtz [Pa13] can be helpful. Paffenholtz's result states that after fixing a dimension $n$ and a small $\varepsilon$ the following set of rational numbers is finite:

$\{\mu \mid \mu>\varepsilon$ and $\mu=\mu(\mathscr{L})$ for an ample line bundle on an $n$-dim $\mathbb{Q}$-Gorenstein toric variety $\}$.

\section{REFERENCES}

[BN08] V.V. Batyrev and B. Nill, Integer points in polyhedra, In Matthias Beck and et. al., editors, Combinatorial aspects of mirror symmetry, volume 452 of Contemp. Math., pp. 35-66. AMS, 2008.

[BS94] M.C. Beltrametti and A.J. Sommese, Some effects of the spectral values on reductions., Classification of algebraic varieties (L'Aquila, 1992), volume 162 of Contemp. Math., pp. 31-48. Amer. Math. Soc., Providence, RI, 1994.

[CC07] R. Curran and E. Cattani, Restriction of A-discriminants and dual defect toric varieties, J. Symbolic Comput. 42 (2007), no. 1-2, 115-135, DOI 10.1016/j.jsc.2006.02.006.

[DDRP12] A. Dickenstein, S. Di Rocco, and R. Piene, Higher order duality and toric embeddings, to appear in Annales de l'Institut Fourier.

[DN10] A. Dichenstein and B. Nill, A simple combinatorial criterion for projective toric manifolds with dual defect, Mathematical Research Letters, 17: 435-448, 2010.

[DS02] A. Dickenstein and B. Sturmfels, Elimination theory in codimension 2, J. Symbolic Comput. 34 (2002), no. 2, 119-135, DOI 10.1006/jsco.2002.0545.

[CCDDRS] E. Cattani, M.A. Cueto, A. Dickenstein, S. Di Rocco, and B. Strumfels, Mixed discriminants, to apper an Math Z.

[DFS07] A. Dickenstein and E. M. Feichtner, Tropical discriminants, J. Amer. Math. Soc. 20 (2007), no. 4, 1111-1133.

[CDR08] C. Casagrande and S. Di Rocco, Projective $\mathbb{Q}$-factorial toric varieties covered by lines, Commun. Contemp. Math. 10 (2008), no. 3, 363-389.

[DS02] A. Dickenstein and B. Sturmfels, Elimination theory in codimension 2, J. Symbolic Comput. 34 (2002), no. 2, 119-135.

[DiR06] S. Di Rocco, Projective duality of toric manifolds and defect polytopes, Proc. London Math. Soc.

(3) 93 (2006), no. 1, 85-104. 
[DDRP12] A. Dickenstein, S. Di Rocco, and R. Piene, Higher order duality and toric embeddings, to appear in Annales de l'Institut Fourier.

[DDRP09] A. Dickenstein, S. Di Rocco, and R. Piene, Classifying smooth lattice polytopes via toric fibrations, Adv. Math. 222 (2009), no. 1, 240-254.

[DRHNP13] S. Di Rocco, B. Nill, C. Haase, and A. Paffenholz, Polyhedral Adjunction Theory, Algebra and Number Theory (to appear).

[EW] G. Ewald, Combinatorial convexity and algebraic geometry, Graduate Texts in Mathematics, vol. 168, Springer-Verlag, New York, 1996.

[Fuj90] T. Fujita, Classification Theories of Polarized Varieties, Lecture Note Ser., London Math. Soc,Cambridge Univ. Press, 155, 1990.

[FU] W. Fulton, Introduction to toric varieties, Annals of Mathematics Studies, vol. 131, Princeton University Press, Princeton, NJ, 1993. The William H. Roever Lectures in Geometry.

[GKZ] I.M. Gel'fand, M.M. Kapranov, and A. Zelevinsky, Discriminants, resultants, and multidimensional determinants, Mathematics: Theory \& Applications, Birkhäuser Boston Inc., Boston, MA, 1994.

[GH79] P. Griffiths and J. Harris, Algebraic geometry and local differential geometry, Ann. Sci. École Norm. Sup. (4) 12 (1979), no. 3, 355-452.

[HNP09] C. Haase, B. Nill, and S. Payne, Cayley decompositions of lattice polytopes and upper bounds for $h^{*}$-polynomials, J. Reine Angew. Math. 637 (2009), 207-216.

[Ito12] A. Ito, Algebro-geometric characterization of Cayley polytopes, arXiv:1202.6663.

[LM00] Antonio A. Lanteri and R. Mallavibarrena, Higher order dual varieties of generically k-regular surfaces, Arch. Math. (Basel) 75 (2000), no. 1, 75-80, DOI 10.1007/s000130050476.

[L94] J.M. Landsberg, On second fundamental forms of projective varieties, Invent. Math. 117 (1994), no. 2, 303-315, DOI 10.1007/BF01232243.

[MT11] Y. Matsui and K. Takeuchi, A geometric degree formula for A-discriminants and Euler obstructions of toric varieties, Adv. Math. 226 (2011), no. 2, 2040-2064, DOI 10.1016/j.aim.2010.08.020.

[ODA] Tadao Oda, Convex bodies and algebraic geometry-toric varieties and applications. I, Algebraic Geometry Seminar (Singapore, 1987), 1988, pp. 89-94.

[Pa13] A. Paffenholz, Finiteness of the polyhedral Q-codegree spectrum, arXiv:1301.4967 [math.CO].

Sandra Di Rocco, Department of Mathematics, Royal institute of Technology (KTH), 10044 STOCKHOLM, SWEDEN

E-mail address: dirocco@kth.se

URL: www.math.kth.se/ dirocco 